\title{
Physical properties of amorphous silicon-carbon alloys produced by different techniques
}

\author{
A. Carbone, F. Demichelis, and G. Kaniadakis \\ Dipartimento di Fisica del Politecnico di Torino, Corso Duca degli Abruzzi 24, 10129 Torino, Italy \\ G. Della Mea and F. Freire \\ Dipartimento di Ingegneria dei Materiali, Universitá di Trento e Unitá, I.N.F.N. Padova, Italy \\ P. Rava \\ Elettrorava S.p.A. 10040 Savonera, Torino, Italy
}

(Received 25 April 1990; accepted 16 August 1990)

Results of a study of compositional, optical, electrical, and structural properties of hydrogen amorphous silicon carbide $(a-\mathrm{SiC}: \mathrm{H})$ prepared, respectively, by glow-discharge (GD) and reactive sputtering (SP) techniques at power densities varying between $1.25 \cdot 10^{-2}$ and $1.25 \cdot 10^{-1} \mathrm{~W} \cdot \mathrm{cm}^{-2}$ for GD samples are presented. Measurements are reported on the composition, optical and IR spectroscopy, and on the temperature dependence of electrical conductivity. All experimental observations suggest that the power density only slightly affects the physical properties of GD silicon-rich samples, whereas those of the carbon-rich SP samples depend more strongly on this deposition parameter. Finally, it is shown that the GD technique can provide films with better characteristics, whereas samples of similar composition prepared by sputtering have higher compositional disorder and are more inhomogeneous.

\section{INTRODUCTION}

Hydrogenated amorphous silicon carbon alloys $(a-\mathrm{SiC}: \mathrm{H})$ are of considerable interest for application in multijunction thin-film solar cells ${ }^{1}$ and in large area white luminescent devices. ${ }^{2}$ These alloys can be prepared by Plasma Enhanced Chemical Vapor Deposition (PECVD), also known as glow-discharge (GD), or by reactive sputtering (SP).$^{3-5}$ In the GD technique, a mixture of silane and a hydrocarbon gas such as methane, ethylene, or tetramethylsilane gases is used; good quality material with a low density of states can be achieved in a $\mathrm{SiH}_{4}+\mathrm{CH}_{4}$ gas mixture. Sputtered $a-\mathrm{SiC}: \mathrm{H}$ samples were found to have electronic properties inferior to those produced by the GD technique. ${ }^{6}$ However, material properties can be controlled better by adjusting deposition parameters in sputtering than in glow-discharge. Among the various preparation parameters, such as gas pressure, substrate temperature, system geometry, etc., the power density can be considered an important parameter since generally the power regime is different between the GD and the SP techniques.

It has been shown ${ }^{3,7}$ that it is possible in the low power regime to separate, in the GD technique, the chemistry of the plasma and that of deposition and prepare $a-\mathrm{SiC}: \mathrm{H}$ films with low densities of states from $\mathrm{SiH}_{4}+\mathrm{CH}_{4}$ mixtures. This paper presents a study of $a-\mathrm{SiC}: \mathrm{H}$ samples of similar composition deposited by the GD technique in $\mathrm{SiH}_{4}+\mathrm{CH}_{4}$ gas mixtures and by the SP technique of a silicon target in $\mathrm{Ar}+\mathrm{CH}_{4}$ gas mixture, respectively, varying the power density. The results obtained on the two sets of samples were compared in order to understand the influence of the power density on film properties.

\section{EXPERIMENTAL}

Two sets of samples were deposited, respectively, by glow-discharge decomposition of $\mathrm{SiH}_{4}+\mathrm{CH}_{4}$ and by sputtering. The deposition parameters are reported in Table I. Samples are labeled GD and SP when obtained, respectively, by glow-discharge or sputtering, followed by a number indicating the total power in watts during the deposition.

The elemental composition was obtained by means of nuclear measurements. A direct analysis of the hydrogen profiles has been made by using the technique of elastic recoil detection analysis (ERDA) of $2.2 \mathrm{MeV}$ ${ }^{4} \mathrm{He}^{+}$ions. The content of carbon and silicon and con-

TABLE I. Deposition conditions.

\begin{tabular}{|c|c|c|}
\hline & GD samples & SP samples \\
\hline Power density $\left(\mathrm{W} \cdot \mathrm{cm}^{-2}\right)$ & $0.0125-0.125$ & $0.6-1.6$ \\
\hline Substrate temperature $\left({ }^{\circ} \mathrm{C}\right)$ & 200 & 200 \\
\hline Growth rate $\left(\AA \cdot \mathrm{s}^{-1}\right)$ & 3.3 & 3.5 \\
\hline Total pressure $(\mathrm{Pa})$ & 40 & 1.6 \\
\hline $\mathrm{CH}_{4}$ flux $(\mathrm{sccm})$ & 115 & 1.5 \\
\hline $\mathrm{SiH}_{4}$ flux (sccm) & 80 & $\cdots$ \\
\hline $\mathrm{H}_{2}$ flux $(\mathrm{sccm})$ & 80 & $\cdots$ \\
\hline Ar flux (sccm) & $\cdots$ & 100 \\
\hline
\end{tabular}


centrations of impurities were obtained by Rutherford backscattering (RBS) measurements.

Optical transmissivity and reflectivity measurements were carried out by a Perkin-Elmer UV-visibleNIR Lambda 9 spectrophotometer. The extraction of the optical constants was done following the procedure described in Ref. 8. IR spectroscopy measurements were made in the absorption mode using a Bruker spectrophotometer between 400 and $4000 \mathrm{~cm}^{-1}$. The ESR spectra were taken on a Varian EPR-019 spectrometer. The spin density was determined by calibration against a standard sample DPPH (1,1 dyphenil -2- pyrylhydrazil) and Varian pitch.

The temperature dependence of the dark current was measured by two-point technique under a vacuum $(\approx 5 \mathrm{~Pa})$ with a Hewlett Packard high resistance meter (MODEL 4329 A).

\section{RESULTS AND DISCUSSION}

In Table II the elemental composition as measured by ERDA and RBS measurements is reported. For the GD samples a very good uniformity of composition was observed for all the samples independent of the power density. In both cases the carbon content is lower than the silicon content. However, in SP samples the hydrogen content is about the same as carbon and the ratio $\mathrm{Si} / \mathrm{C}$ is lower than for GD samples. The optical constants were obtained from transmittance and reflectance measurements. In Table III the thicknesses, the values of Tauc's gap $E_{g}$, and the index of refraction $n$ $(\lambda=1.5 \mu \mathrm{m})$ for GD and SP samples are reported. A

TABLE II. Elemental composition parameters.

\begin{tabular}{lccc}
\hline \hline Sample & Si/C & H/C & Ar/C \\
\hline GD & 5.7 & 3.3 & $\ldots$ \\
SP100 & 2.2 & 1.3 & 0.12 \\
SP150 & 2.6 & 0.9 & 0.12 \\
SP200 & 2.8 & 0.9 & 0.12 \\
SP250 & 2.8 & 0.9 & 0.14 \\
\hline
\end{tabular}

TABLE III. Optical and electrical properties.

\begin{tabular}{lcccc}
\hline \hline Sample & $\begin{array}{c}\text { Thickness } \\
(\mu \mathrm{m})\end{array}$ & $\begin{array}{c}E_{g} \\
(\mathrm{eV})\end{array}$ & $\begin{array}{c}n \\
(\lambda=1.5 \mu \mathrm{m})\end{array}$ & $\begin{array}{c}N_{s} \\
\left(\mathrm{spin} \cdot \mathrm{cm}^{-3}\right)\end{array}$ \\
\hline GD020 & 0.42 & 1.89 & 3.11 & $1.4 \cdot 10^{19}$ \\
GD030 & 0.43 & 1.99 & 3.16 & $1.2 \cdot 10^{19}$ \\
GD100 & 0.50 & 2.07 & 3.23 & $9.0 \cdot 10^{18}$ \\
GD150 & 0.53 & 2.06 & 3.30 & $8.4 \cdot 10^{18}$ \\
GD200 & 0.58 & 2.07 & 3.40 & $7.7 \cdot 10^{18}$ \\
SP100 & 0.45 & 2.02 & 2.71 & $1.2 \cdot 10^{20}$ \\
SP150 & 0.58 & 1.89 & 2.85 & $9.0 \cdot 10^{19}$ \\
SP200 & 0.71 & 1.79 & 3.00 & $6.0 \cdot 10^{19}$ \\
SP250 & 0.78 & 1.69 & 3.12 & $1.2 \cdot 10^{20}$ \\
\hline \hline
\end{tabular}

slight increase of the optical gap $E_{g}$ can be observed up to a saturation value in GD and a monotonic decrease in SP samples. The SP samples show a variation of $19 \%$ for $E_{g}$ and $14 \%$ for $n$, respectively, when the power density changes from 0.6 to $1.6 \mathrm{~W} / \mathrm{cm}$. On the contrary, the GD samples show for both $E_{g}$ and $n$ a variation of $9 \%$. The values of $E_{g}$ found in this work are in agreement with that of Refs. 5 and 9. For the SP samples, having the same hydrogen content, the decrease of $E_{g}$ could be related to a higher concentration of voids when the power density increases, in agreement with the literature. ${ }^{10}$ For GD samples, they are known to be more homogeneous; the increase of $E_{g}$ indicates that carbon is present in the diamond-like fourfold coordination when the deposition power increases. ${ }^{11}$ This behavior couldn't be attributed to hydrogen content, because it is constant for all the samples (Table II). The refractive index, $n$, of GD samples confirms the values of the $\mathrm{Si} / \mathrm{C}$ ratio as obtained by nuclear measurements. In fact, the index of refraction approximates to the values of amorphous silicon, whereas the $n$ value of SP samples approximates to that of carbon, in agreement with the $\mathrm{Si} / \mathrm{C}$ ratio for these samples. ${ }^{4}$

The IR absorption coefficient has been deduced from the measured IR transmission spectra, taking into account the interference fringes and absorption due to the silicon substrate. A very different width of the peaks can be observed in SP and GD samples. In the band peaked at $2100 \mathrm{~cm}^{-1}$ a wide peak can be attributed to a doublet at 2000 and $2100 \mathrm{~cm}^{-1}$, according to Brodsky et al. ${ }^{12}$ and Freeman et al. ${ }^{13}$ while one due to $\mathrm{SiH}$ and $\mathrm{SiH}_{2}$ stretching vibrations, respectively, is resolved in GD samples only. In the band between 600 and $1100 \mathrm{~cm}^{-1}$, GD samples show a finely resolved structure, as can be seen in Fig. 1(a), where the IR absorption spectra of GD samples obtained with $100 \mathrm{~W}$ power are shown. In Fig. 1(b) the IR absorption spectra of SP samples obtained with $100 \mathrm{~W}$ power are reported. The peak centered at $1000 \mathrm{~cm}^{-1}$ can be attributed to $s p^{3}$ bonds in the $\mathrm{CH}_{2}$ configuration, ${ }^{14}$ and the peak at $780 \mathrm{~cm}^{-1}$ is due to rocking or bond-bending vibrations of $\mathrm{CH}_{3}$ radicals bonded to $\mathrm{Si}$. The peak at $650 \mathrm{~cm}^{-1}$ is attributed to the $\mathrm{SiH}$ bond-bending mode. ${ }^{15}$ The weak structures present in GD spectra do not appear resolved in SP spectra. This fact indicates that these samples are compositionally inhomogeneous.

The integrated intensities $S$ of the different peaks are defined as

$$
S=\int \frac{\alpha(\omega)}{\omega} d \omega
$$

where $\alpha(\omega)$ is the absorption coefficient for frequency $\omega$ and the integration is performed over the deconvoluted peak corresponding to the vibration mode. For GD samples the integrated intensities, $S$, of all the vibration 


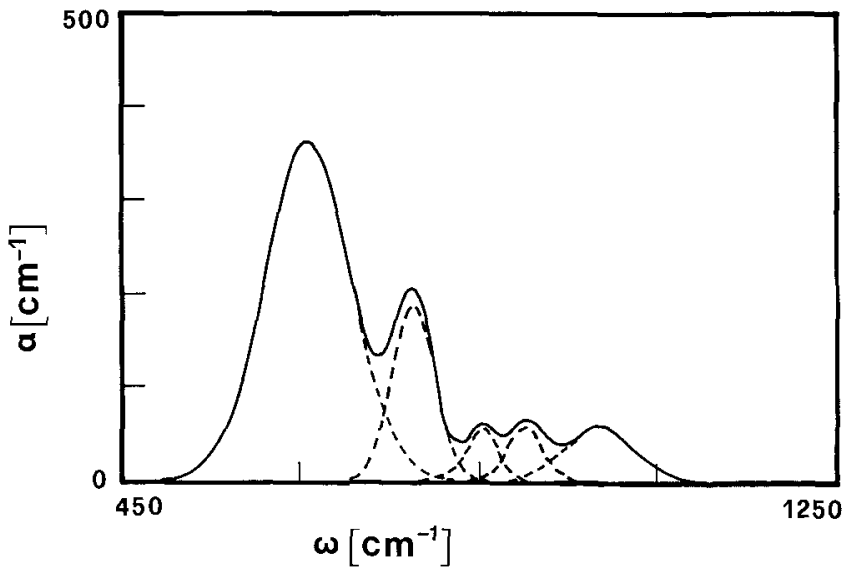

(a)

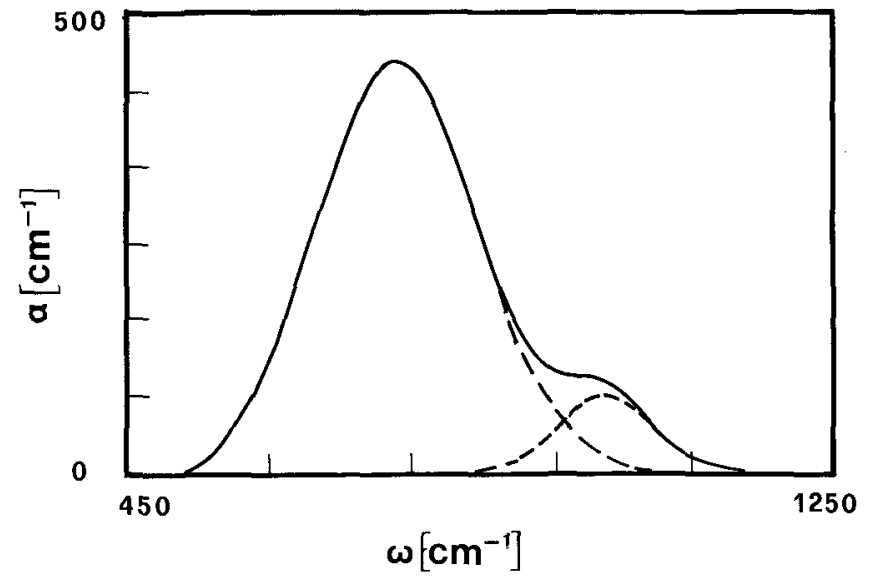

(b)

FIG. 1. (a) IR absorption spectra for GD100 sample. (b) IR absorption spectra for SP100 sample.

modes $\left(\mathrm{SiH}_{2}, \mathrm{SiH}, \mathrm{CH}_{2}\right)$ are, within experimental errors, independent of the deposition power density. These results are consistent with the samples being of uniform composition, as deduced by nuclear measurements. Figure 2 shows the absorption-integrated intensities of the vibrational modes versus the power for SP samples. It can be observed that the trend of the $\mathrm{CH}_{2}$ vibrational mode has about the same behavior as the elemental composition trend (carbon and hydrogen). The same considerations seem to be extended to the $\mathrm{SiH}_{2}$ and $\mathrm{CSiH}_{3}$ vibrational modes. These results suggest that the bonds of hydrogen with silicon and carbon for SP samples do not depend on deposition power density, and therefore the power density has no effect on the integrated infrared absorption. A comparison of the amplitude of ESR signals to the signal of standard material allows us to obtain the density of spins $N_{s}$. For GD samples a slow decrease of $N_{s}$ from $7.7 \cdot 10^{18}$ to $1.4 \cdot 10^{19}$ spin $\cdot \mathrm{cm}^{-3}$ can be observed as the power den-

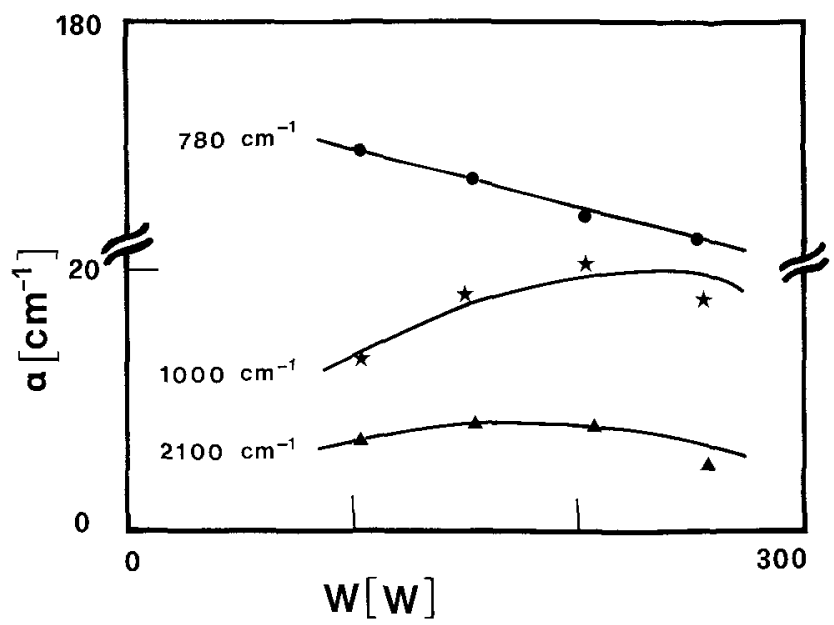

FIG. 2. Absorption integrated intensities of the vibrational modes versus the power for SP samples. sity increases from $1.25 \cdot 10^{-2}$ to $1.25 \cdot 10^{-1} \mathrm{~W} \cdot \mathrm{cm}^{-2}$. For SP samples, the values of $N_{s}$ show no significant differences as the power density changes. Their values are of the order of about $10^{20} \mathrm{spin} \cdot \mathrm{cm}^{-3}$, one order of magnitude higher than for GD samples. This can be attributed to the presence of inhomogeneities and a high compositional disorder, probably due to the higher deposition power density in the sputtering technique, which leads to different chemical decomposition of $\mathrm{CH}_{4} \cdot{ }^{16}$

The temperature dependence of dark conductivity for GD and SP samples is shown in Figs. 3(a) and 3(b), respectively, where a plot of $\log \sigma_{D}$ against $1000 / T$ is reported. It can be observed that the nonlinear plot of $a-\mathrm{CSi}: \mathrm{H}$ films is generally described in terms of continuous curvature, in agreement with earlier results appearing in the literature. ${ }^{5}$ The behavior of curves is consistent with conduction taking place by phonon assisting hopping. It is indeed known ${ }^{5}$ that in sputtered $a-\mathrm{SiC}: \mathrm{H}$ material this conduction mechanism predominates at all the temperatures.

The SP samples show during warming a different trend of $\log \sigma_{D}$ in the low temperature regime and the same one in the high temperature regime (above $T \approx 373 \mathrm{~K}$ ) and during the cooling process. In these samples a higher carbon content is present, and in these carbon-rich films the graphitic-like threefold coordination predominates, giving higher conductivity. The asdeposited films having different elemental composition show different conductivity, indeed, in the low temperature regime. The warming probably produces structural variation acting on the inhomogeneities and voids present in sputtered samples up to the temperature $T=373 \mathrm{~K}$; after that all the samples have the same behavior in the high temperature region and in the cooling curve.

The trend of conductivity of the GD samples is the same for all the samples during warming, because the as-deposited films have essentially the same composi- 


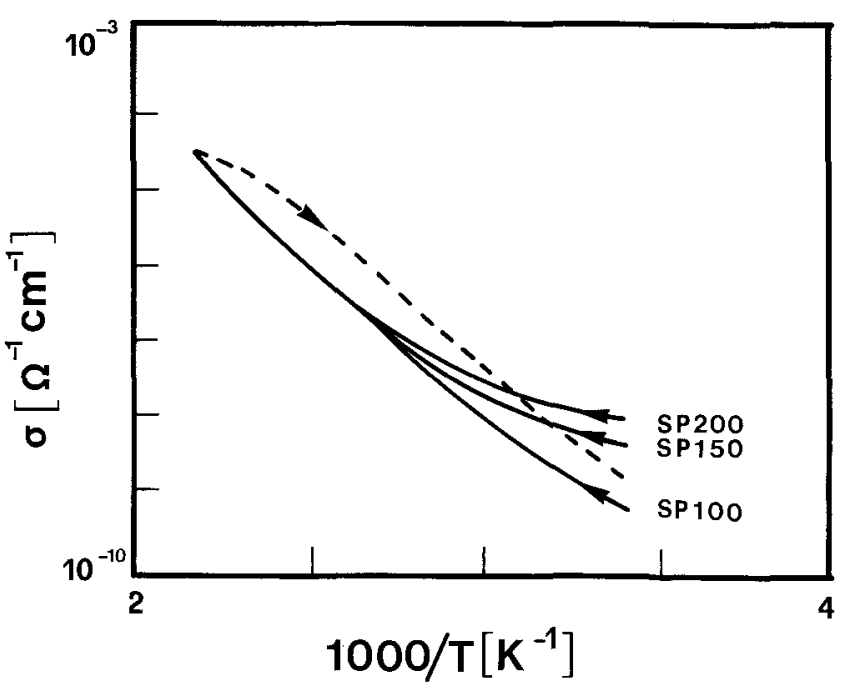

(a)

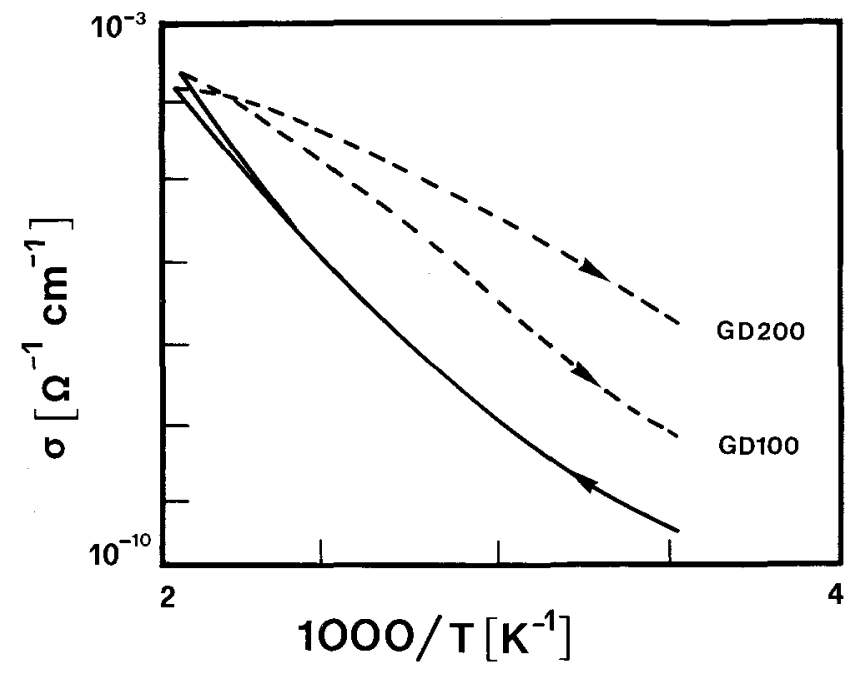

(b)

FIG. 3. (a) Temperature dependence of dark conductivity for GD samples. (b) Temperature dependence of dark conductivity for SP samples.

tion (Table II), with the other physical properties varying slowly with the deposition conditions (Table III). On the contrary, the behavior of conductivity in the cooling is different for the various GD samples; a tentative interpretation can be the following. The elemental composition of GD films indicates that they are siliconrich samples. Anderson and Spear ${ }^{5}$ suggest that in such samples, at high temperature, a fraction of the excess carbon in SiC tends to form a threefold coordinated graphitic environment, leading to an increase of conductivity. This suggestion agrees with the present results which indicate also that for higher power density the carbon-rich region predominates. This fact could justify the high conductivity of the samples at room temperature and the hopping conduction mechanism.

The temperature dependence of the conductivity shows the substantial difference of behavior of the two sets of GD and SP samples. This confirms that the physical properties of $a-\mathrm{SiC}: \mathrm{H}$ films strongly depend upon the deposition technique as well as the deposition conditions.

\section{CONCLUSIONS}

The main results obtained in our study on the elemental composition and the optical, electrical, and structural properties of films prepared by glow discharge and sputtering under similar conditions as a function of the power density may be summarized as follows.

(1) The elemental composition of silicon-rich GD samples as well as structural properties are practically independent on the power density. A difference is observed in the conductivity during cooling, in the optical properties and in the density of spins.
(2) The carbon-rich SP samples also show a difference in elemental composition: the silicon content decreases as the power density increases. The structural properties as deduced from the IR measurements are in agreement with the composition. The optical gap decreases as the power density increases, indicating a more graphitic structure for high power density. Such results are confirmed by the trend of conductivity versus the warming temperature. The density of spins indicates high compositional disorder in the samples.

(3) By comparing the results of characterization measurements made on the two different types of $a-$ $\mathrm{CSi}: \mathrm{H}$ films, it can be deduced that the properties of the GD samples show a slow dependence on the power density, while the SP sample properties depend more strongly on this parameter.

\section{ACKNOWLEDGMENT}

This work was partially supported by Consiglio Nazionale delle Ricerche (PFE2).

\section{REFERENCES}

${ }^{1}$ Y. Kuwano, M. Ohnishi, H. Nishiwaski, S. Tsuda, T. Fukatsu, K. Enomoto, Y. Nakashima, and H. Tazni, 16th IEEE PV Spec Conference, San Diego, CA (IEEE, New York, 1982), p. 1331.

${ }^{2}$ H. Munekata and H. Kukimoto, Appl. Phys. Lett. 42, 432 (1983).

${ }^{3}$ J. Pezzin, I. Solomon, B. Bourdon, J. Fontenille, and E. Ligeon, Thin Solid Films 62, 327 (1979).

${ }^{4}$ M. P. Schmidt, J. Bullot, M. Gauthier, P. Cordier, I. Solomon, and H. Tran-Quoc, Philos. Mag. B 51, 581 (1985).

${ }^{5}$ D. A. Anderson and W. E. Spear, Philos. Mag. B 35, 1 (1977).

${ }^{6}$ J. Bullot and M. P. Schmidt, Phys. Status Solidi (B) 143, 345 (1987).

${ }^{7}$ M. P. Schmidt, I. Solomon, H. Tran-Quoc, and J. Bullot, J. NonCryst. Solids 77-78, 849 (1985). 
${ }^{8}$ F. Demichelis, G. Kaniadakis, A. Tagliaferro, and E. Tresso, Appl. Opt. 26, 1717 (1987).

${ }^{9}$ A. H. Mahan, B. von Roedern, D. L. Williamson, and A. Madan, J. Appl. Phys. 57, 8 (1985).

${ }^{10}$ W. Paul and D. Anderson, Solar Energy Materials 5, 229 (1981).

${ }^{11}$ J. Robertson, Adv. Phys. 35, 4, 317 (1986).

${ }^{12}$ M. H. Brodsky, M. Cardona, and J. J. Cuomo, Phys. Rev. B 16, 3556 (1977).
${ }^{13}$ E. C. Freeman and W. Paul, Phys. Rev. B 18, 4288 (1978).

${ }^{14}$ B. Dishler, Proc. 7th Int. Symp. on Plasma Chemistry (Eindhoven, July 1985), Vol. I, p. 45.

${ }^{15}$ F. Demichelis, G. Kaniadakis, E. Mezzetti, P. Mpawenayo, A. Tagliaferro, E. Tresso, P. Rava, and G. Della Mea, Nuovo Cimento 9D, 393 (1987).

${ }^{16}$ T. D. Moustakas, Semiconductors and Semimetals, edited by J. I. Pankove (Academic Press, New York, 1984), Vol. 21, p. 55. 